\title{
Study of Efficacy of Cilostazole in Peripheral Arterial Occlusive Disease
}

\author{
Sattar Khan ${ }^{1}$, Divakar Shenoy ${ }^{2}$ \\ ${ }^{1}$ Resident In General Surgery, 2. Professor Of Surgery, K.S.Hegde Medical Academy \\ “Devaru Doddavanu” Building $2^{\text {nd }}$ Cross Saptapur Dharwad Karnataka India
}

\begin{abstract}
Peripheral arterial occlusive disease (PAOD), commonly referred to as peripheral arterial disease $(P A D)$ or peripheral vascular disease (PVD), refers to the obstruction or deterioration of blood flow through the arteries, exclusive of the coronary and intracranial vessels. ${ }^{1}$ The incidence of symptomatic PAD increases with age, from approximately $0.3 \%$ / year for men aged 40 to 55 years to approximately 1\%/year for men older than 75 years. ${ }^{2}$ Peripheral arterial occlusive disease is an important manifestation of atherosclerosis involving the arteries of legs. ${ }^{3}$ Vascular surgeons continue to encounter complications of atherosclerosis as their most common clinical challenge. ${ }^{4}$ This observational study was conducted with patient receiving $100 \mathrm{mg}$ Cilostazole twice daily and assessing the claudication distance at the end of 3months. There was statistically significant increase in the claudication distance in patients receiving Cilostazole. We concluded Cilostazol is an effective drug in increasing claudication distance in patients with peripheral vascular occlusive disease.
\end{abstract}

Keywords: peripheral arterial occlusive disease, intermittent claudication, claudication distance.

\section{Introduction}

Peripheral arterial occlusive disease (PAOD), commonly referred to as peripheral arterial disease (PAD) or peripheral vascular disease (PVD), refers to the obstruction or deterioration of blood flow through the arteries, exclusive of the coronary and intracranial vessels. ${ }^{1}$ The incidence of symptomatic PAD increases with age, from approximately $0.3 \%$ / year for men aged 40 to 55 years to approximately $1 \%$ /year for men older than 75 years. $^{2}$ Peripheral arterial occlusive disease is an important manifestation of atherosclerosis involving the arteries of legs. ${ }^{3}$ Vascular surgeons continue to encounter complications of atherosclerosis as their most common clinical challenge. ${ }^{4}$ Although only $1-2 \%$ of people are affected from intermittent claudication before 50 yrs of age, it increases to 5\% in those aged 50 -60 yrs and 10\% in those older than 70yrs. ${ }^{5}$ PAD is more prevalent in non-white population, and this is not completely explained by an increased incidence of co-morbid diseases. ${ }^{6}$ Risk is increased in smokers and in patients with hypertension (HTN), dyslipidemia, hypercoagulable states, renal insufficiency \& diabetes mellitus (DM). The prevalence of PAD is strikingly higher in a younger diabetic population, affecting one in three diabetics older than 50yr. The risk of PAD also increases in individuals who are older than $50 \mathrm{yr}$, males, obese and with a family history of vascular disease, myocardial infarction (MI) or cerebral vascular accident (CVA). Other risk factors that are being studied include levels of various inflammatory mediators, such as C-reactive protein and homocysteine. ${ }^{2}$ Intermittent claudication(IC) / claudication pain is defined as "cramping, aching, fatigue, or any patient-reported pain in one or both legs that occurred during walking that was sufficient to cause the subject to reduce the pace or cease ambulation". Money S R and others conducted a double blind, multi-centric, randomized, placebo controlled trail on effect of cilostazol on walking distance in patients with intermittent claudication caused by peripheral vascular disease (239 patients).Study showed significant increase in absolute claudication distance in patients treated with cilostazole $(47 \%$ increase) compared with placebo( $12.9 \%$ increase $)$ with $\mathrm{p}<0.001 .^{7}$ Robless $\mathrm{P}$ and others conducted a double-blind, RCT of cilostazole versus placebo. Seven RCT trails were included. The weighted mean difference (WMD) for initial claudication distance was improved by $31.1 \mathrm{~m}$ with $95 \%$ confidence interval(CI) in patients treated with $100 \mathrm{mg}$ BD dose. The WMD for initial claudication distance was improved by $41.3 \mathrm{~m}$ with $95 \% \mathrm{CI}$ in patients treated with $50 \mathrm{mg}$ BD dose. ${ }^{8}$ Travis M Falconer and others conducted a metaanalyses which suggested that cilostazol increases maximum walking distance by $40 \%-50 \%$ and improves other objective measures of walking. ${ }^{9}$

\section{Methodology}

This hospital based observational study was conducted at Justice K S Hegde hospital, Mangalore, Karnataka, India. It was done after obtaining ethical committee approval from September 2014 to September 2016. Patients who meet the inclusion criteria were assessed for pain free walking distance on a treadmill with a speed of $4 \mathrm{~km} / \mathrm{hr}$. Then patients were started on cilostazole $100 \mathrm{mg}$ BD. Patients were assessed before starting cilastozole and at the end of 3 month for the pain free walking distance (PFWD). 


\section{Inclusion criteria-}

- Patients presenting with features suggestive of chronic lower limb arterial occlusive disease i.e claudication pain

- Patients age more than 30yrs.

Exclusion criteria-

- Patients with peripheral arterial disease of regions other than the lower extremities.

- Patients presenting with gangrene.

- Patients not willing to give consent.

- Patients who have undergone surgical intervention.

- Patients with unstable coronary artery disease, symptomatic cardiac arrhythmias.

- Patients lost for follow-up.

Statistical analysis was carried out by using SPSS software and p-value was calculated using student-t test.

\section{Results}

A total of 127 patients were assessed in the study. 23 patients were excluded from the study as 22 patients were lost for follow up and 1 patient developed adverse effect (headache) after starting the drug. Hence a total of 104 patients were included in the study, of which 91 were males and 13 were females(Graph 1). Most of the patients included in the study were in the range of 51 to $70 \mathrm{yrs}(\mathrm{Graph} 2)$.

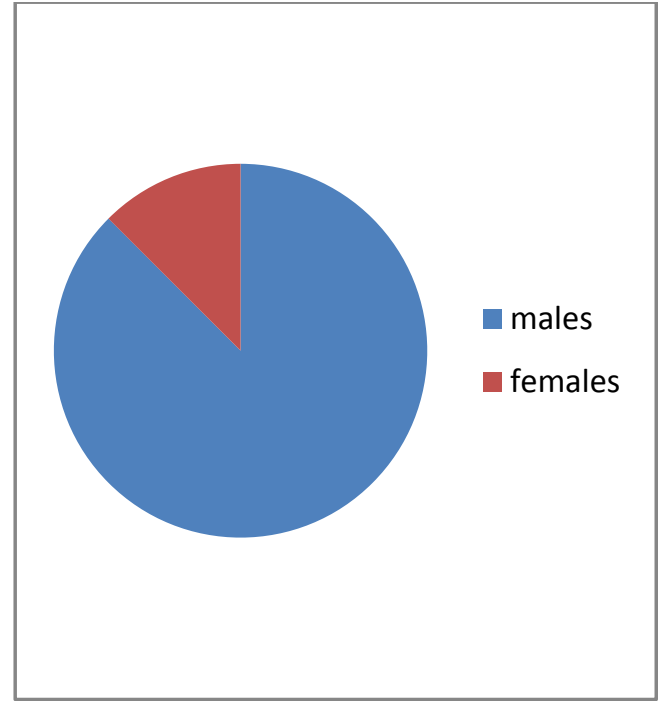

Graph 1

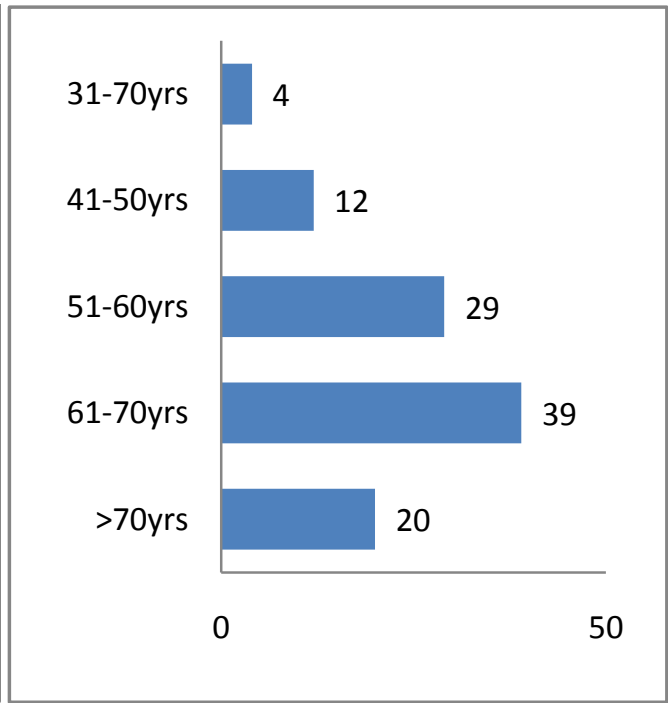

Graph 2

In the present study 39 patients were diabetic and 52 patients gave history of smoking(pack year>10). Mean claudication distance before starting the drug was 92meters, which increased to 132 meters at the end of 3 months with an increase in maen value by 1.43 times with a p-value of $<0.01$.

\section{Conclusion}

After analyzing the data, its conclusive that cilostazol is effective in increasing claudication distance in patients with peripheral vascular occlusive disease. In the present observational study, the mean increase in the pain free walking distance was 1.43 times (i.e. $43 \%$ increase with a p-value of $<0.01$ ) the initial distance. In the present study most of the patients were in the age group of 51-70yrs $(65 \%)$ and most of them were male $(87.5 \%)$. Also 39 patients $(37.5 \%)$ were diabetic and 52 patients $(50 \%)$ were smokers. All the patients with history of smoking had discontinued smoking during the study period. Cilostazol was discontinued in one patient due to headache which was not controlled with the use of analgesics. Cilostazol is well tolerated in most of the patients with side effect incidence, $<2 \% .{ }^{27}$ Hence we concluded Cilostazol is an effective drug in increasing claudication distance in patients with peripheral vascular occlusive disease.

Ethical approval: "All procedures performed in studies involving human participants were in accordance with the ethical standards of the institutional ethics committee."

Informed consent: Informed consent was obtained from all the participants included in the study. 


\section{References}

[1]. Ouriel K. Detection of peripheral arterial disease in primary care. JAMA 2001 sep;286:1380-1.

[2]. Sabiston textbook of surgery, The biological basis of modern surgical practice, $19^{\text {th }}$ edition.

[3]. Hiatt WR. Medical treatment of peripheral arterial disease and claudication. N Eng J Med 2001;344:380-1.

[4]. De Palma RG. Atherosclerosis: Pathology, pathogenesis and medical management, $6^{\text {th }}$ ed. Chapter 6. In: Moore WS, editor. Vascular surgery: A Comprehensive Review. Philadelphia: WB Saunders Company;2002.p91-04.

[5]. Nehler MR, Taylor LM, Moneta GL, Porter JM. Natural history and non-operative treatment of chronic lower extremity ischemia. $6^{\text {th }}$ ed. Chapter 15. In: Moore WS, editor. Vascular surgery: A Comprehensive Review. Philadelphia: WB Saunders Company;2002.p 264-75.

[6]. Criqui MH, Vargas V, Denenberg JO, et al: Ethinicity and peripheral arterial disease: The San Diego Population syudy. Circulation 112:2703-2707, 2005

[7]. Effect of cilostazole on walking distances in patients with intermittent claudication caused by peripheral vascular disease. Money SR, Herd JA, Isaacsohn JL, Davidson M, Cutler B, Heckman J , Forbes WP. J Vasc Surg. 1998 Feb;27(2):267-74; discussion 274-5.

[8]. Cilostazole for PAD. Robless P, Mikhailidis DP, Stansby GP. Cochrane Database syst Rev. 2008 jan 23;(1);CD003748.

[9]. Management of peripheral arterial disease in the elderly: focus on cilostazol by Travis M Falconer, John W Eikelboom, Graeme J Hankey, Paul E Norman at School of Surgery, University of Western Australia, Fremantle Hospital, Western Australia. Clinical Interventions in Aging 2008:3(I) 17-23. 